\title{
Tomographic Study of Femoral Positioning in Anterior Cruciate Ligament Reconstruction Using the Transtibial Technique
}

\author{
Robson Rocha da Silva, $\mathrm{MD}^{1}$, Marcos Almeida Matos, $\mathrm{PhD}^{2}$, Valonie Carlos Neves Brasileiro Costa, $\mathrm{MD}^{1}$, \\ Vitor Hugo Abreu Azevedo de Morais, $\mathrm{MD}^{3}$, and Luiz Eduardo Lago de Castro, $\mathrm{MD}^{3}$ \\ ${ }^{1}$ Department of Orthopedics, Hospital Santa Izabel, Salvador; ${ }^{2}$ Department of Pos-Graduation, Bahiana School of Medicine and Public Health, Salvador; ${ }^{3}$ Department \\ of Radiology, Hospital Santa Izabel, Salvador, Brazil
}

\begin{abstract}
Purpose: To analyze the location of the femoral tunnel by three-dimensional computed tomography (3D CT) of the lateral condyle in patients who underwent arthroscopic single-bundle anterior cruciate ligament (ACL) reconstruction, performed using a modified transtibial technique, and to compare the results with data from the literature.

Materials and Methods: Seventeen patients with ACL lesions underwent modified transtibial ACL reconstruction. Postoperatively, 3D CT examinations were performed and the images were analyzed by the quadrant system described by Bernard and Hertel to define the femoral tunnel coordinates.

Results: The mean value of femoral tunnel location coordinates was $37.1 \pm 5.9$ in the horizontal plane and $23.9 \pm 7.5$ in the vertical plane. Compared with other studies using the transtibial or transportal technique, femoral positioning was improved in the vertical plane. The outside-in technique provided the best results both in the vertical and horizontal planes.

Conclusions: The modified transtibial technique was not effective for systematically anatomic femoral tunnel positioning; however, it was relatively better than the conventional transtibial technique.
\end{abstract}

Keywords: Knee, Anterior cruciate ligament reconstruction, Three-dimensional computed tomography, Transtibial technique, Tunnel position

\section{Introduction}

Anterior cruciate ligament (ACL) reconstruction is one of the most frequently performed orthopedic surgery procedures at present $^{1)}$. Over the last two decades, the transtibial (TT) reconstruction technique where the femoral tunnel is created through

Received January 19, 2016; Revised (1st) November 25, 2016; (2nd) March 28, 2017; Accepted April 28, 2017

Correspondence to: Robson Rocha da Silva, MD

Department of Orthopedics, Hospital Santa Izabel, Praca Conselheiro Almeida Couto, 500, Nazare, Salvador-BA, 40050-410, Brazil

Tel: +55-71-2203-8069, Fax: +55-71-2203-8069

E-mail: robroc@superig.com.br

Source of funding: The study was conducted at the Orthopedic department of Hospital Santa Izabel.

This is an Open Access article distributed under the terms of the Creative Commons Attribution Non-Commercial License (http://creativecommons.org/licenses/by-nc/4.0/) which permits unrestricted non-commercial use, distribution, and reproduction in any medium, provided the original work is properly cited. the tibial tunnel has established itself as a fast, reproducible and easily performed method ${ }^{2,3}$. This technique rapidly attained wide acceptance, and is extensively used with the publication of good results being attributed to it.

However, recent studies have offered improved knowledge of the ACL anatomy, revealing that conventional techniques, such as the TT type, do not provide replication of the original ligament insertions ${ }^{2,4)}$. Especially in the TT technique, the tibial tunnel dictates the femoral tunnel, which often results in a vertical graft orientation. Vertical grafts exhibit inferior rotational control, which results in secondary arthritis in a long-term follow-up. In recent years, more anatomical tunnel placement has been proven to provide superior results in biomechanical and cadaveric studies $^{5-7)}$. Therefore, changes in the surgical paradigm toward a more anatomic positioning of the tunnels have led to the introduction of new reconstruction techniques that seek to restore articular biomechanics more precisely, approximating the knee to its original form ${ }^{8)}$. 
In addition to proposing new techniques, studies have suggested changes in the TT technique, with the intention of improving the positioning of the femoral tunnel ${ }^{3)}$. These changes are based on accurate identification of the ligament insertions and femoral guide rotation in the posterior part of the lateral femoral condyle by placing a guidewire as close as possible to the anatomic point. Many surgeons who have utilized the new techniques in ACL reconstruction have reported good clinical results; however, more evidence is required to determine the superiority of the new techniques to the modified transtibial (MTT) technique.

In the past, the femoral tunnel position was compared in cadaver studies or in others using conventional radiographs inappropriate for adequate assessment of the position of the tunnels ${ }^{4,9,10)}$. At present, the sites for graft fixation have been extensively evaluated by means of three-dimensional computed tomography (3D $\mathrm{CT}$ ) images of the femoral condyle using the quadrant method described by Bernard and Hertel- ${ }^{2-4,9,11}$.

The primary aim of this study was to evaluate and measure the position of the femoral tunnel by means of 3D CT images in a series of patients who underwent reconstruction of the ACL using the MTT technique. We compared the coordinates of the femoral fixation site obtained in this study with the ideal anatomic positioning described in the literature and with the results in other studies using the TT technique or other more anatomic techniques, with use of the same method of evaluation.

\section{Materials and Methods}

This was an exploratory research in a series of patients from a reference hospital for knee surgery, selected by means of the nonprobabilistic, sequential sampling technique in the period from April 2013 to November 2014. All patients were operated on by the same surgeon for anatomic reconstruction of the ACL with a single-bundle, hamstring autograft using the same MTT reconstruction technique. Fixation on the femoral side was randomly performed with the RigidFix ST (DePuy Mitek, Raynhan, MA,
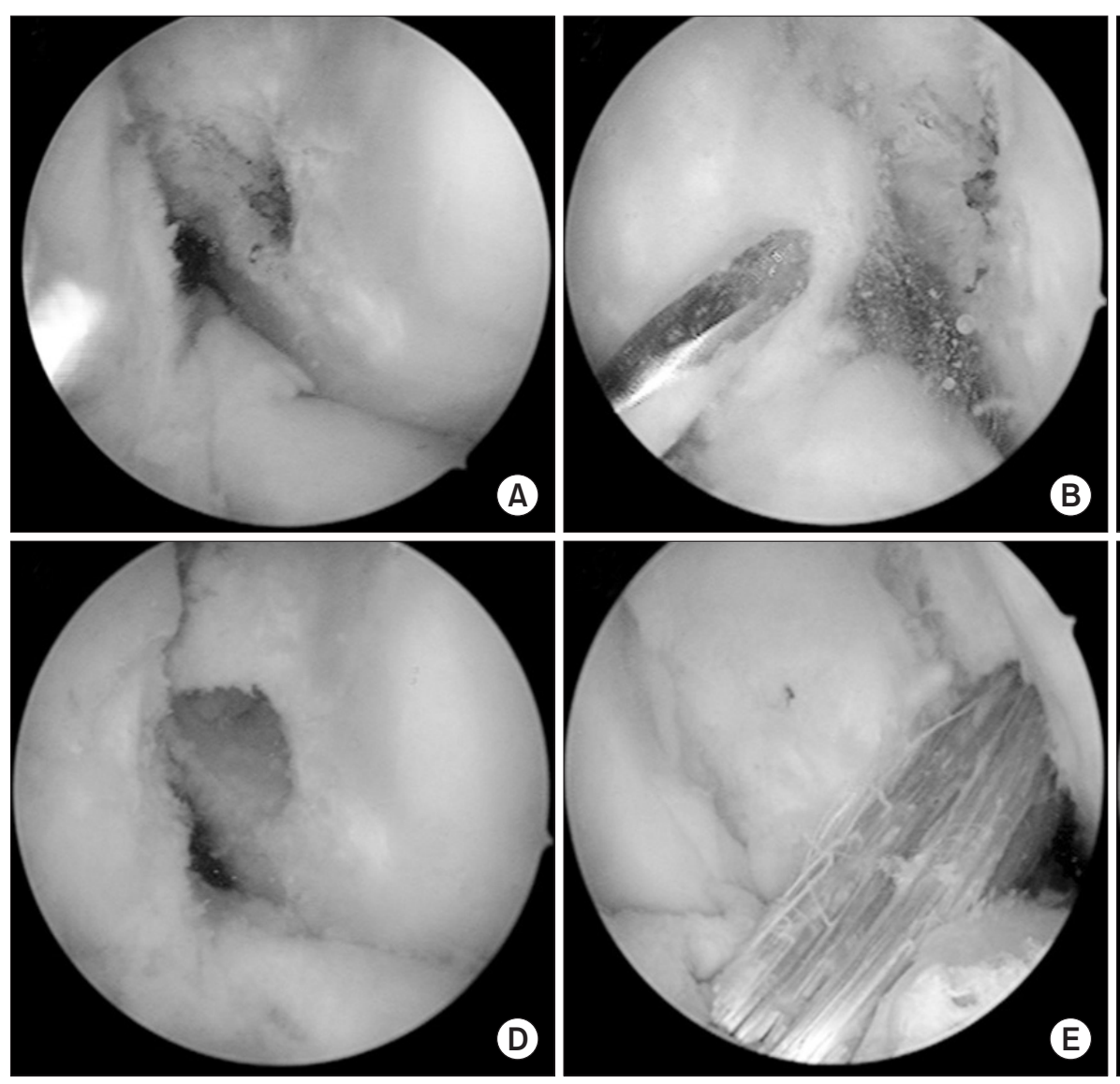

Fig. 1. Arthroscopic surgery images for anterior cruciate ligament (ACL) reconstruction by the modified Transtibial Technique. (A) View of the ACL footprint from the anteromedial portal. (B) View of the tibial guidewire from the anterolateral portal. (C)View of the burr guidewire with femoral guide from the anterolateral portal. (D) View of the femoral tunnel from the anteromedial portal. (E) View of the graft from the anterolateral portal. (F) View of the graft from the anteromedial portal. 
USA), Retroboton (Arthrex, Naples, FL, USA) or Transfix (Arthrex) system.

The following inclusion criteria were applied: patients aged 18 to 60 years and primary ACL reconstruction for an isolated ACL lesion without associated ligamentous injuries. The exclusion criteria were as follows: tomographic changes in the lateral femoral condyle anatomy (arthrosis, notchplasty, revision surgery, or sequelae resulting from fractures or infection), interruption in follow-up, or unavailability for postoperative 3D CT.

Sixty ACL reconstructions were performed by the surgeon during the study period. Twenty-two patients did not fulfill the inclusion criteria, 6 patients were excluded because they presented with changes in the 3D CT, and 15 patients were excluded from the study because they did not maintain follow-up or did not have the 3D CT performed.

\section{Surgical Technique}

After harvesting the grafts, the arthroscopic procedure began by sequentially inspecting the articulation. Then, reconstruction of the ACL was performed using the MTT technique.

\section{1) Identification of the femoral footprint}

As opposed to the traditional TT technique that uses the clock reference to construct the femoral tunnel, in the MTT technique, with the arthroscope inserted into the medial portal, the remaining stumps of the anteromedial and posterolateral fiber bundles were located, and an intermediate point was marked between the two bands that served as a reference for tunnel positioning, using either microfracture or radiofrequency (Fig. 1A).

\section{2) Positioning of the tibial guide pin}

From the view of the lateral portal, a guide pin was positioned by using an elbow-shaped external tibial guide (Fig. 2), introduced through the medial portal. With the directional angle pointing toward the femoral footprint, the guide pin was positioned so that it would emerge between the spines at a point closest to that of the medial tibial spine and be directed towards a point as close as possible to the place demarcated on the femur. In this main surgical step, certain adjustments were made both in the degree of knee flexion and obliquity of the guide, and some attempts were frequently made until best positioning was achieved (Fig. 1B). Then, the tunnel was drilled over the guide.

\section{3) Positioning of the femoral guide}

With the knee flexion of around 65 degrees, an ACL femoral offset guide, two numbers above the diameter of the graft, was introduced into the tibial tunnel and rotated so that the femoral guide pin would attain the position as close as possible to the place demarcated on the femur (Fig. 1C).

\section{4) Removal of the femoral guide}

To remove the femoral guide, the guide pin was first removed, avoiding trauma to the posterior crossed ligament due to its more inferior and inclined positioning.

Then, the femoral tunnel was constructed. The graft was positioned and fixed by the chosen technique (Fig. 1). The immediate postoperative and rehabilitation protocols were identical in all cases. Discharge from hospital was the second day after surgery.

The mean positioning of the femoral tunnel obtained in this
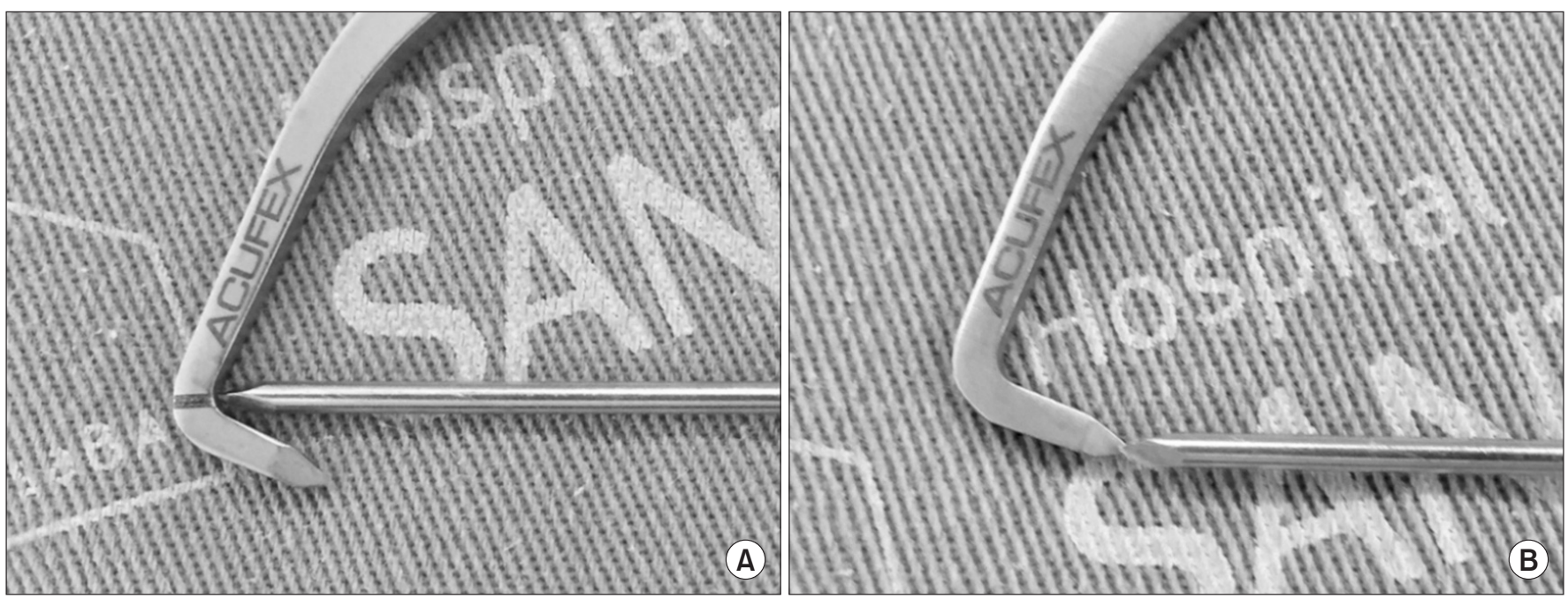

Fig. 2. Models of guide used in this study. (A) Guide ACUFEX Director Elbow Aimer (Smith \& Nephew Endoscopy), (B) ACUFEX Director Tip Aimer (Smith \& Nephew Endoscopy). 

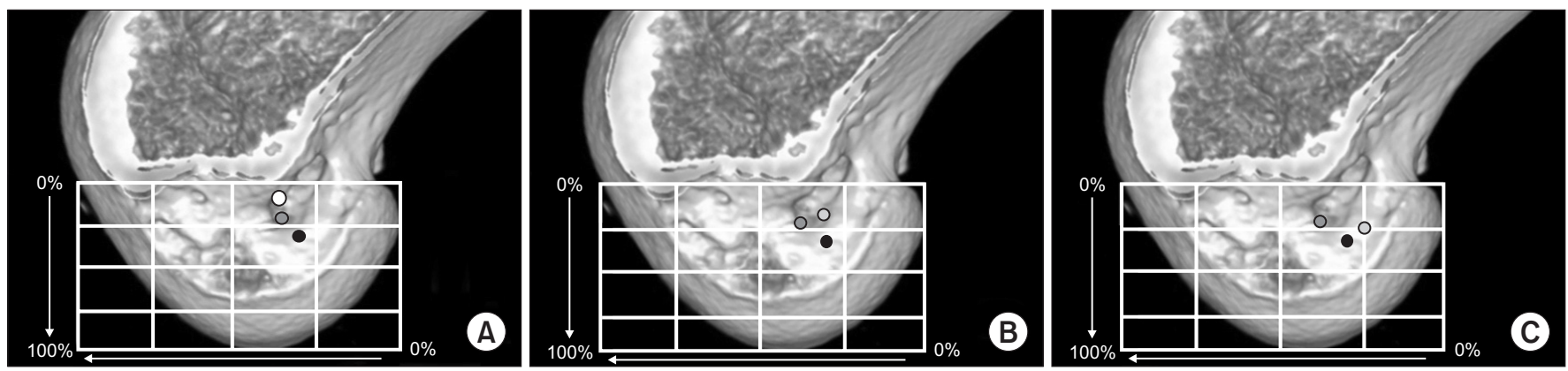

Fig. 3. (A) In white: Mean value of positions of the femoral tunnel in the transtibial technique performed by Kopf et al. ${ }^{2)}(37.2 \times 11.3)$. In dark grey: Mean value of positions of the present study $(37.5 \times 23.5)$. In black: Ideal anatomic position described in the literature by Bird et al. ${ }^{8)}(28.0 \times 35.0)$. $(\mathrm{B})$ In light grey: Mean value of femoral tunnel positions in the transportal technique performed by Bird et al. ${ }^{8)}(30.0 \times 17.0)$. In dark grey: Mean value of positions of the present study $(37.5 \times 23.5)$. In black: Ideal anatomic position described by Bird et al. ${ }^{8)}$ by $(28.0 \times 35.0)$. (C) In light grey: Mean value of femoral tunnel positions in the outside-in technique performed by Ahn et al. (27.0×24.7). In dark grey: Mean value of positions of the present study (37.5×23.5). In black: Ideal anatomic position described in the literature by Bird et al. ${ }^{8)}(28.0 \times 35.0)$.

study by means of 3D CT was compared with that in other studies using the traditional TT technique, the transportal anatomic technique, and the outside-in technique (Fig. 3).

\section{Three-Dimensional Tomographic Analysis}

In the first postoperative month, 3D CT reconstruction of the distal third of the femur was performed with the medial condyle suppressed for perfect visualization of the medial wall of the lateral condyle according to the technique validated by Basdekis et al. ${ }^{10)}$. Using a 128 channel multidetector CT appliance (Somatom Definition, Siemens, Erlangen, Germany), the volumetric images acquired were sent to the workstation (Syngo.via, Siemens) where $3 \mathrm{D}$ reconstruction was carried out.

The standard protocol with the quadrant method suggested by Bernard and Hertel ${ }^{11)}$ was used to determine the position of the center of the femoral tunnel. The grid adjustment was based on the Blumensaat line and the lateral condyle contour, allowing the horizontal and vertical axes to be determined. In the 3D reconstruction images, the central point of the femoral tunnel coordinates was expressed as a percentage (\%) of the dimension (mm) in the quadrant in the posterior to anterior direction (parallel to the Blumensaat line) and from the proximal to distal direction (perpendicular to the Blumensaat line).

\section{Ethical Aspects}

The research project was approved by the Research Ethics Committee of Hospital Santa Izabel da Santa Casa de Misericordia da Bahia. All the individuals selected for the research were informed with regard to the research objectives, its risks and benefits; they were also requested to sign the Term of Free and Informed Consent. The patients were given the right to desist from participa- tion at any moment they wished to do so.

\section{Statistical Analysis}

The results obtained were presented in the form of descriptive statistics using frequency distribution tables for nominal variables and mean \pm standard deviation for continuous variables. The Student $t$-test was used to compare the means of the coordinates in the horizontal and vertical planes with the coordinates of the ideal point for anatomic reconstruction and with the coordinates of the point of reference for TT reconstruction as well as the coordinates of the point obtained in transportal ACL reconstruction, all of which were obtained using the same 3D reconstruction technique as described in the literature ${ }^{2,9}$. The Student $t$ test was also used to compare the means of the coordinates of the present study with the ideal anatomic position and with the means of three different techniques published in the literature (transportal anatomic, TT, and outside-in techniques) obtained by the same 3D CT reconstruction technique ${ }^{2,9)}$. For all statistical analyses, significance was accepted at $\mathrm{p}<0.05$.

\section{Results}

Of the patients selected, 2 were women. The age range was 27 to 54 years. In 7 cases, the right knee was operated. Three femoral fixation methods were used, which are shown together with the other demographic and surgical data in Table 1.

The mean value of the femoral tunnel position coordinates on the $3 \mathrm{D}$ CT reconstruction image was $37.5 \pm 5.7$ in the plane parallel to the Blumensaat line and $23.5 \pm 6.7$ in the vertical plane.

Compared to the data of studies on TT reconstruction ${ }^{2)}$, there was greater approximation to the ideal point with statistically 
Table 1. Demographic Data

\begin{tabular}{|c|c|c|c|c|c|c|}
\hline \multirow{2}{*}{ No. } & \multirow{2}{*}{ Age (yr) } & \multirow{2}{*}{ Sex } & \multirow{2}{*}{ Laterality } & \multirow{2}{*}{ Fixation device } & \multicolumn{2}{|c|}{ CT measurements } \\
\hline & & & & & Vertical & Horizontal \\
\hline 1 & 51 & M & $\mathrm{L}$ & RigidFix ST (Johnson) & 20.00 & 39.00 \\
\hline 2 & 27 & $\mathrm{~F}$ & $\mathrm{R}$ & Retro button (Arthrex) & 20.20 & 44.80 \\
\hline 3 & 36 & M & $\mathrm{R}$ & RigidFix ST (Johnson) & 18.40 & 33.30 \\
\hline 4 & 54 & $\mathrm{~F}$ & $\mathrm{~L}$ & RigidFix ST (Johnson) & 27.39 & 33.30 \\
\hline 5 & 38 & M & $\mathrm{L}$ & Retro button (Arthrex) & 34.78 & 27.30 \\
\hline 6 & 35 & M & $\mathrm{R}$ & Transfix (Arthrex) & 16.88 & 34.70 \\
\hline 7 & 30 & M & $\mathrm{R}$ & RigidFix ST (Johnson) & 20.30 & 43.80 \\
\hline 8 & 49 & M & $\mathrm{R}$ & RigidFix ST (Johnson) & 24.30 & 45.00 \\
\hline 9 & 45 & M & $\mathrm{R}$ & Transfix (Arthrex) & 39.00 & 33.50 \\
\hline 10 & 27 & M & $\mathrm{R}$ & RigidFix ST (Johnson) & 19.00 & 36.72 \\
\hline 11 & 37 & M & $\mathrm{L}$ & Retro button (Arthrex) & 23.18 & 36.50 \\
\hline 12 & 32 & M & $\mathrm{L}$ & Retro button (Arthrex) & 18.57 & 40.00 \\
\hline 13 & 40 & M & $\mathrm{R}$ & RigidFix ST (Johnson) & 22.80 & 33.30 \\
\hline 14 & 25 & M & $\mathrm{R}$ & Retro button (Arthrex) & 22.20 & 41.21 \\
\hline 15 & 45 & M & $\mathrm{L}$ & RigidFix ST (Johnson) & 33.78 & 44.05 \\
\hline 16 & 27 & M & $\mathrm{R}$ & RigidFix ST (Johnson) & 14.75 & 27.40 \\
\hline 17 & 33 & M & $\mathrm{R}$ & RigidFix ST (Johnson) & 19.38 & 43.47 \\
\hline
\end{tabular}

CT: computed tomography. L: left, R: right.

Table 2. Comparison of Computed Tomography (CT) Coordinates of This Study with Coordinates of Transtibial Technique

\begin{tabular}{cccccc}
\hline CT coordinates & This study & Kopf et al. ${ }^{2)}$ & p-value & Ahn et al. $^{3)}$ & p-value \\
\hline Horizontal & $37.5 \pm 5.7$ & $37.2 \pm 5.5$ & 0.838 & $36.5 \pm 7.6$ & 0.485 \\
Vertical & $23.5 \pm 6.7$ & $11.3 \pm 6.6$ & 0.001 & $7.7 \pm 7.2$ & $<0.001$ \\
\hline
\end{tabular}

Values are presented as mean \pm standard deviation.

Table 3. Comparison of Computed Tomography (CT) Coordinates of This Study with Coordinates of Traditional Transportal Technique

\begin{tabular}{ccccc}
\hline CT coordinates & This study & Bird et al. ${ }^{9}$ & p-value & 95\% confidence interval \\
\hline Horizontal & $37.5 \pm 5.7$ & 30.0 & $<0.001$ & 4.5 to 10.4 \\
Vertical & $23.5 \pm 6.7$ & 17.0 & 0.001 & 3.0 to 9.7 \\
\hline
\end{tabular}

Values are presented as mean \pm standard deviation.

Table 4. Comparison of Computed Tomography (CT) Coordinates of This Study with Coordinates of Outside-in Reconstruction

\begin{tabular}{ccccc}
\hline CT coordinates & This study & Ahn et al. ${ }^{3}$ & p-value & $95 \%$ confidence interval \\
\hline Horizontal & $37.5 \pm 5.7$ & $24.7 \pm 4.9$ & $<0.001$ & 9.8 to 15.7 \\
Vertical & $23.5 \pm 6.7$ & $27.0 \pm 7.0$ & 0.044 & -7.0 to -0.10 \\
\hline
\end{tabular}

Values are presented as mean \pm standard deviation.

significant difference in the vertical plane (Table 2). The comparisons in the mean coordinates with the TT reconstruction are presented in Table 3. The comparisons with the outside- in and ideal anatomic reconstruction are presented in Tables 4 and 5, respectively.

The mean position of the femoral tunnel obtained in this study by means of 3D CT and that in TT reconstruction published in the literature were compared with the ideal position. Comparison 
Table 5. Comparison of Computed Tomography (CT) Coordinates of This Study with Coordinates of Ideal Position for Anatomic Reconstruction Defined in the Literature

\begin{tabular}{ccccr}
\hline CT coordinates & This study & Ahn et al. ${ }^{3)}$ & p-value & $95 \%$ confidence interval \\
\hline Horizontal & $37.5 \pm 5.7$ & 28.0 & $<0.001$ & 6.5 to 12.4 \\
Vertical & $23.5 \pm 6.7$ & 35.0 & $<0.001$ & -14.9 to -8.0 \\
\hline
\end{tabular}

Values are presented as mean \pm standard deviation.

of the position of this study with the position of the transportal anatomic technique, outside-in technique and ideal anatomic position are shown in Fig. 3.

\section{Discussion}

In this study, the mean values of the coordinates of the femoral tunnel were obtained by means of 3D CT images in a series of ACL reconstruction performed with a MTT technique in order to create a more anatomic femoral tunnel. The mean femoral positioning in our patients more approximated the optimal anatomic point defined in the literature ${ }^{9)}$ than that obtained by means of the TT technique in other publications ${ }^{2}$.

In the absence of standardized methods to obtain the images for assessment of femoral tunnel position both in the horizontal and vertical planes ${ }^{12)}$, the quadrant method applicable to $3 \mathrm{D} C \mathrm{CT}$ models has been referred to as one of the best ways to evaluate the femoral tunnel position and thus it has been widely used in previous studies ${ }^{10)}$. Using this method, Bird et al. ${ }^{9)}$ reported the coordinates of the optimal anatomic femoral tunnel position, which was based on the mean coordinates previously described by six authors. Other studies also reported on the femoral tunnel coordinates in transportal anatomic ACL reconstrcution ${ }^{9)}$ and ACL reconstruction using the outside-in and TT techniques ${ }^{2}$. In this study, we made comparisons with other techniques in terms of the anatomic point in ACL reconstruction.

In our study, despite the modifications made to the TT technique, it was not possible to replicate the original position either in the parallel or perpendicular plane to the Blumensaat line as documented in the current literature ${ }^{13-15)}$. Previous studies presented the difficulty of the TT technique attaining the anatomic femoral position, which could also limit rotational stability ${ }^{3,16)}$. Kopf et al. ${ }^{4)}$ also reported that TT reconstruction resulted in nonanatomically positioned tunnels in a 3D CT assessment.

Compared to the conventional transportal ACL reconstruction $^{9)}$, statistically significant differences were observed in the two planes in our study. In the parallel plane to the Blumensaat line, the difference favored the transportal reconstruction, which, on average, better attained the anatomic point of reference. In the transversal plane, the difference favored our method. In other words, the femoral position observed in our patients approximated the optimal anatomic position. Bird et al. ${ }^{9)}$, in a study comparing the femoral tunnel position between the traditional transportal technique and a modified anatomic technique, observed that the conventional method produced, in the transversal plane, a femoral tunnel closer to the Blumensaat line, and attributed that finding to the difficulty of precisely identifying the points of reference of the femoral footprint by the arthroscopic method. In our cases, a better positioning in the vertical plane was limited by both the femoral guide on the over-the-top and the tibial tunnel direction where, despite the maneuvers in the degree of knee flexion as well as the external rotation of the guide, it was not possible to position the femoral guide at a lower point. In the horizontal plane, the mistake could have been caused by both the previous reasons mentioned and the difficulty in identifying the optimal point as referred by Bird et al. ${ }^{9}$.

On the comparison of our data with the mean values of coordinates mentioned in studies where the TT technique was used $^{2,3)}$, there was no significant difference in the plane parallel to the Blumensaat line, whereas, in the plane perpendicular to the Blumensaat line, we attained a better position. In spite of the fact that these authors referred to the practice of rotating the femoral guide on the over-the-top, no improvement in the position of the femoral tunnel was achieved. Our results were probably obtained due to the adequate positioning of the tibial tunnel (to direct the graft to an anatomic position) with the external rotation of the femoral guide. This more meticulous positioning of the tibial tunnel allows the graft to be aligned with the axis of the original ligament; nonetheless, this position is hardly reproducible. Other studies have pointed out that, for an appropriately selected tibial tunnel, a more anatomic femoral tunnel may be attained by positioning the graft more horizontally than in the classical TT techniques ${ }^{17)}$. Hosseini et al. ${ }^{17)}$ confirmed this proposition. From the biomechanical engineering point of view, the femoral tunnel location in the TT technique depends on the position of the tibial tunnel. Thus, they suggested that in 3D magnetic resonance imaging, the orientation of the graft in the articular space would be affected in the coronal plane by its position in the mediolateral 
direction, and in the sagittal plane by the anteroposterior posi$\operatorname{tion}^{17)}$. In cases of failure, the orientation of the graft was significantly more vertical, especially in the sagittal plane.

This study demonstrated that the positioning of the femoral tunnel and consequently of the graft in the TT technique can be approximated to the anatomic position, mainly in the transversal plane. The anatomic technique of outside-in reconstruction much better approximated the ideal positioning ${ }^{3)}$.

The TT technique was considered the "gold standard" for reconstructing ACL lesions and, due to its reproducibility, it was widely used a few years ago, ${ }^{9,18)}$. As it has been shown that the technique does not reproduce the ACL anatomy, the attempt to improve it should be made ${ }^{19-23)}$. All clinical and functional inferiority attributed to the TT technique has been explained by a very important factor: the non-anatomic positioning of the femoral tunnel. This was considered the key focal point of our study, and thus our patients were operated by using an MTT technique for the purpose of improving positioning. More importantly, we compared our results with not only what was considered the ideal but also with those of other techniques including the conventional TT technique.

We observed some limitations of our study. The fact that all surgeries were performed by the same surgeon limits extrapolation of the data because they are not representative of a common exercise but of an individual experience. The localization of the anatomic point during the surgery as well as the localization of the center of the femoral tunnel and the lack of standardization in obtaining 3D CT are the possible sources of bias in this study. Furthermore, the absence of correlation with clinical data and lack of follow-up do not allow us to infer whether the differences observed in the study are significant and have an impact on articular stability.

\section{Conclusions}

From these data, we could conclude that we attained better positioning than that found in the conventional TT techniques; nevertheless, we did not attain the ideal position described in the literature. We could attribute this improvement in position to the rotational maneuver applied to the femoral guide combined with the improved direction of the tibial tunnel to obtain a femoral tunnel closer to the original anatomy.

\section{Conflict of Interest}

No potential conflict of interest relevant to this article was re- ported.

\section{References}

1. Buoncristiani AM, Tjoumakaris FP, Starman JS, Ferretti M, $\mathrm{Fu} \mathrm{FH}$. Anatomic double-bundle anterior cruciate ligament reconstruction. Arthroscopy. 2006;22:1000-6.

2. Kopf S, Forsythe B, Wong AK, Tashman S, Anderst W, Irrgang JJ, Fu FH. Nonanatomic tunnel position in traditional transtibial single-bundle anterior cruciate ligament reconstruction evaluated by three-dimensional computed tomography. J Bone Joint Surg Am. 2010;92:1427-31.

3. Ahn JH, Jeong HJ, Ko CS, Ko TS, Kim JH. Three-dimensional reconstruction computed tomography evaluation of tunnel location during single-bundle anterior cruciate ligament reconstruction: a comparison of transtibial and 2-incision tibial tunnel-independent techniques. Clin Orthop Surg. 2013;5:26-35.

4. Kopf S, Forsythe B, Wong AK, Tashman S, Irrgang JJ, Fu FH. Transtibial ACL reconstruction technique fails to position drill tunnels anatomically in vivo 3D CT study. Knee Surg Sports Traumatol Arthrosc. 2012;20:2200-7.

5. Franceschi F, Papalia R, Rizzello G, Del Buono A, Maffulli N, Denaro V. Anteromedial portal versus transtibial drilling techniques in anterior cruciate ligament reconstruction: any clinical relevance? A retrospective comparative study. Arthroscopy. 2013;29:1330-7.

6. Koutras G, Papadopoulos P, Terzidis IP, Gigis I, Pappas E. Short-term functional and clinical outcomes after ACL reconstruction with hamstrings autograft: transtibial versus anteromedial portal technique. Knee Surg Sports Traumatol Arthrosc. 2013;21:1904-9.

7. Mulcahey MK, David TS, Epstein DM, Alaia MJ, Montgomery KD. Transtibial versus anteromedial portal anterior cruciate ligament reconstruction using soft-tissue graft and expandable fixation. Arthroscopy. 2014;30:1461-7.

8. Dienst M, Burks RT, Greis PE. Anatomy and biomechanics of the anterior cruciate ligament. Orthop Clin North Am. 2002;33:605-20,

9. Bird JH, Carmont MR, Dhillon M, Smith N, Brown C, Thompson P, Spalding T. Validation of a new technique to determine midbundle femoral tunnel position in anterior cruciate ligament reconstruction using 3-dimensional computed tomography analysis. Arthroscopy. 2011;27:1259-67.

10. Basdekis G, Christel P, Anne F. Validation of the position of the femoral tunnels in anatomic double-bundle ACL recon- 
struction with 3-D CT scan. Knee Surg Sports Traumatol Arthrosc. 2009;17:1089-94.

11. Bernard M, Hertel P. Intraoperative and postoperative insertion control of anterior cruciate ligament-plasty: a radiologic measuring method (quadrant method). Unfallchirurg. 1996; 99:332-40.

12. Fernandes TL, Martins NMMF, Watai FA, Neto CA, Pedrinelli A, Hernandes AJ. Tomografia computadorizada 3D para mensuracao do posicionamento femoral na reconstrucao do LCA. Acta Ortop Bras. 2015;23:11-5.

13. Bedi A, Musahl V, Steuber V, Kendoff D, Choi D, Allen AA, Pearle AD, Altchek DW. Transtibial versus anteromedial portal reaming in anterior cruciate ligament reconstruction: an anatomic and biomechanical evaluation of surgical technique. Arthroscopy. 2011;27:380-90.

14. Dargel J, Schmidt-Wiethoff R, Fischer S, Mader K, Koebke J, Schneider T. Femoral bone tunnel placement using the transtibial tunnel or the anteromedial portal in ACL reconstruction: a radiographic evaluation. Knee Surg Sports Traumatol Arthrosc. 2009;17:220-7.

15. Pinczewski LA, Salmon LJ, Jackson WF, von Bormann RB, Haslam PG, Tashiro S. Radiological landmarks for placement of the tunnels in single-bundle reconstruction of the anterior cruciate ligament. J Bone Joint Surg Br. 2008;90:172-9.

16. Howell SM, Deutsch ML. Comparison of endoscopic and two-incision techniques for reconstructing a torn anterior cruciate ligament using hamstring tendons. Arthroscopy.
1999;15:594-606.

17. Hosseini A, Lodhia P, Van de Velde SK, Asnis PD, Zarins B, Gill TJ, Li G. Tunnel position and graft orientation in failed anterior cruciate ligament reconstruction: a clinical and imaging analysis. Int Orthop. 2012;36:845-52.

18. Asagumo H, Kimura M, Kobayashi Y, Taki M, Takagishi K. Anatomic reconstruction of the anterior cruciate ligament using double-bundle hamstring tendons: surgical techniques, clinical outcomes, and complications. Arthroscopy. 2007;23:602-9.

19. Heming JF, Rand J, Steiner ME. Anatomical limitations of transtibial drilling in anterior cruciate ligament reconstruction. Am J Sports Med. 2007;35:1708-15.

20. Cohen SB, Fu FH. Three-portal technique for anterior cruciate ligament reconstruction: use of a central medial portal. Arthroscopy. 2007;23:325.

21. Getelman MH, Friedman MJ. Revision anterior cruciate ligament reconstruction surgery. J Am Acad Orthop Surg. 1999;7:189-98.

22. Piasecki DP, Bach BR Jr, Espinoza Orias AA, Verma NN. Anterior cruciate ligament reconstruction: can anatomic femoral placement be achieved with a transtibial technique? Am J Sports Med. 2011;39:1306-15.

23. Steiner ME, Battaglia TC, Heming JF, Rand JD, Festa A, Baria M. Independent drilling outperforms conventional transtibial drilling in anterior cruciate ligament reconstruction. Am J Sports Med. 2009;37:1912-9. 